\title{
Adaptation des parcours d'usinage au processus de fabrication UGV cas de l'ébauche des moules
}

\author{
Emmanuel Duc ${ }^{1, a}$, Serge Segura ${ }^{2}$, Vincent Pateloup $^{3}$ et Thomas Razier ${ }^{4}$ \\ 1 LaMI, Institut Français de Mécanique Avancée, Campus des Cézeaux, BP 265, 63175 Aubière Cedex, France \\ 2 Dassault Systèmes Provence, 53 avenue de l'Europe, 13082 Aix-en-Provence Cedex 2, France \\ 3 Institut Universitaire de Technologie, Allée André Maurois, 87100 Limoges, France \\ ${ }^{4}$ Institut Français de Mécanique Avancée, Campus des Cézeaux, BP 265, 63175 Aubière Cedex, France
}

Reçu le 15 mars 2007, accepté le 6 juillet 2007

\begin{abstract}
Résumé - Cette communication présente une étude sur l'adaptation des trajets d'usinage au processus de fabrication par UGV. La définition d'un processus performant passe par la sélection conjointe d'une stratégie d'usinage et de conditions de coupe associées. Lors de la programmation d'un trajet d'usinage, le programmeur doit choisir une stratégie d'usinage et un mode de calcul des trajets adapté à la pièce à réaliser et qui n'introduit pas de phénomènes perturbateurs sur les éléments clés de la productivité du processus de fabrication : le comportement cinématique de la machine et le comportement mécanique de l'outil. Pour réduire l'influence de ces phénomènes, les éditeurs de FAO proposent de nombreuses solutions et options. Il devient alors difficile de choisir et de paramétrer une opération d'usinage. Après avoir analysé l'ensemble des difficultés posées par l'usinage de ce type de pièce, nous présentons une classification des problèmes. Selon le besoin, cette grille d'analyse facilite le choix de la stratégie d'usinage.
\end{abstract}

Mots clés : FAO / conditions de coupe / calcul de trajets d'usinage / comportement cinématique des machines / comportement mécanique des outils / ébauche

\begin{abstract}
Tool path adaptation for HSM manufacturing mold and die roughing. This paper presents a study of the tool path adaptation to the HSM machining process. The definition of an efficient process imposes the common selection of the milling method and of the cutting conditions. Concerning the tool path programming, the user must choose a milling method and an algorithm dedicated to the part to be machined. The computed tool path does not introduce a reduction of the productivity by appearance of perturbations on the kinematical behaviour of the machine tool and on the mechanical behaviour of the tool. To reduce the influence of the perturbations, CAM software proposes many solutions and options. It is then difficult to choose and to define a machining operation. After an analysis of difficulties due to such part, we present a problem classification. According to the requirement, such classification simplifies the choice of the machining method.
\end{abstract}

Key words: CAM / cutting conditions / kinematical machine tool behaviour / mechanical tool behaviour / roughing

\section{Introduction}

L'industrie des moules et matrices et plus généralement des pièces manufacturées cherche toujours à réduire les coûts de production et par conséquence à diminuer les temps d'usinage. Ces coûts sont directement liés aux performances du processus de fabrication qui repose, en particulier, sur la CFAO et l'usinage à grande vitesse (UGV). Les exigences de qualité ainsi que les nécessités économiques actuelles imposent dorénavant que

\footnotetext{
a Auteur pour correspondance : emmanuel.duc@ifma.fr
}

tous les éléments intervenant dans le processus de fabrication soient optimisés de façon globale.

Tout particulièrement, les performances intrinsèques de l'opération d'ébauche sont directement liées aux performances en situation réelle de production des outils et des machines utilisées mais également des parcours d'usinage calculés. En effet, la trajectoire d'usinage la mieux adaptée à un usinage dépend à la fois des capacités de l'outil, de la dynamique de la machine, de la matière usinée mais, également et surtout, de la géométrie de la zone usinée. Il n'y a pas, a priori, une stratégie d'usinage 
unique idéalement adaptée à tous les types d'usinage, car une légère modification des conditions d'emploi recommandées peut remettre totalement en cause la méthode d'usinage choisie.

La programmation de tels usinages est particulièrement complexe. En effet, il n'est pas toujours simple, voire possible de choisir la stratégie la mieux adaptée au besoin. Le programmeur est dépendant du logiciel utilisé. Avant de procéder au calcul, il doit choisir une stratégie d'usinage parmi une large offre de modules et renseigner un grand nombre de paramètres, dont le lien avec l'objectif fixé n'est pas clairement défini. Ainsi, le programmeur doit posséder un niveau d'expertise suffisant sur le logiciel utilisé afin de trouver rapidement la meilleure solution. Enfin, dans le cas où le trajet outil généré n'est pas satisfaisant, il n'y a pas de méthodologie simple et efficace pour l'améliorer.

L'objet de cette communication est alors de présenter une analyse permettant de guider les choix du programmeur par la définition de classes d'emploi des différentes stratégies proposées et de l'aider à paramétrer les systèmes de FAO.

À partir de l'analyse du comportement de l'outil en usinage dans différents matériaux et du comportement de la machine en suivi de trajectoires, nous proposons plusieurs critères facilitant le choix et l'évaluation de la stratégie d'usinage la mieux adaptée. Ces critères permettent de comparer les différentes stratégies d'usinage entre elles et d'en mesurer les performances avant l'usinage. Le programmeur peut alors conclure sur la pertinence technologique des trajets par rapport aux objectifs visés par le processus de fabrication en se référant aux critères globaux quantifiés.

Ainsi, nous montrons la cohérence entre les différentes solutions proposées et la variété des besoins des utilisateurs.

Cette communication est structurée de la manière suivante. Dans un premier temps, nous conduisons une analyse de la cellule d'usinage pour identifier les problèmes posés par les trajets d'usinage. Nous en déduisons alors les contraintes à respecter par les trajets d'usinage pour obtenir le meilleur comportement possible de la cellule d'usinage. Enfin, nous montrons comment cette étude peut permettre au programmeur de choisir une stratégie d'usinage et de valider ses décisions avant la fabrication.

\section{2 Étude comportementale de la cellule d'usinage}

L'opération d'ébauche des pièces manufacturées est particulièrement exigeante pour le système d'usinage que ce soit au niveau de l'outil ou de la machine outil. En effet, l'opération d'ébauche est souvent longue et coûteuse sans pour autant apporter de la valeur ajoutée au produit final. Il convient donc de la réduire le plus possible. Cela se traduit au niveau de l'usinage par la recherche de conditions de coupe toujours plus élevées, que ce soit sur les vitesses d'avance ou sur les profondeurs de passes axiale et radiale. Ces conditions engendrent alors de grandes vitesses

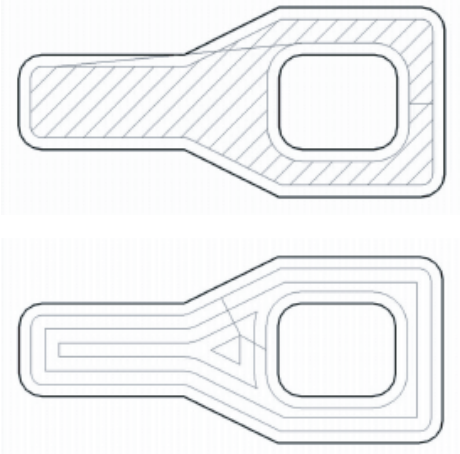

Fig. 1. Usinage par aller et retour et usinage hélicoïdal d'une poche à îlot.

de déplacement, de forts débits copeaux et une puissance de coupe consommée importante.

Par contre, la recherche de la productivité et l'augmentation des conditions de coupe entraînent deux effets contraignants :

- la diminution de la robustesse du processus d'usinage car une faible variation autour des conditions programmées peut engendrer un accident d'usinage coûteux,

- la diminution de la fiabilité du processus qui permet de garantir le respect des conditions de coupe requises au niveau du programme de FAO et au niveau du suivi de la trajectoire en temps réel.

Il est donc nécessaire de procéder à une évaluation globale de la performance de l'ensemble du processus. Pour cela, les trajets d'usinage calculés doivent être adaptés à la cellule d'usinage et engendrer un mouvement de l'outil satisfaisant, qui sollicite la cellule de manière la plus productive possible sans accident. Ainsi, au niveau du calcul du trajet, il est nécessaire d'étudier l'influence des paramètres de programmation de la FAO sur le comportement de la machine. Cette étude permet d'extraire des règles de construction des trajets qui doivent être respectées par les systèmes de FAO.

Dans la suite de cette section, nous allons initialement analyser les trajets d'usinage dédié à l'ébauche des moules. Puis nous étudions le comportement de la machine et de l'outil pendant l'usinage, sollicités par de tels parcours.

\section{1 Étude géométrique des trajets d'usinage}

L'ébauche des moules produit des trajets dont la géométrie est bien connue. La figure 1 présente les deux méthodes d'usinage, usuellement définies dans la littérature. La première concerne le balayage par aller et retour d'une zone, alors que la seconde est un usinage en colimaçon ou hélicoïdal qui permet de respecter le mode de coupe $[1,2]$.

L'usinage de ce type de zone impose une plongée de l'outil dans la matière, selon une pente et un mouvement donnés. De plus, l'usinage est souvent réalisé en plusieurs passes jointes par des mouvements de liaison entre 


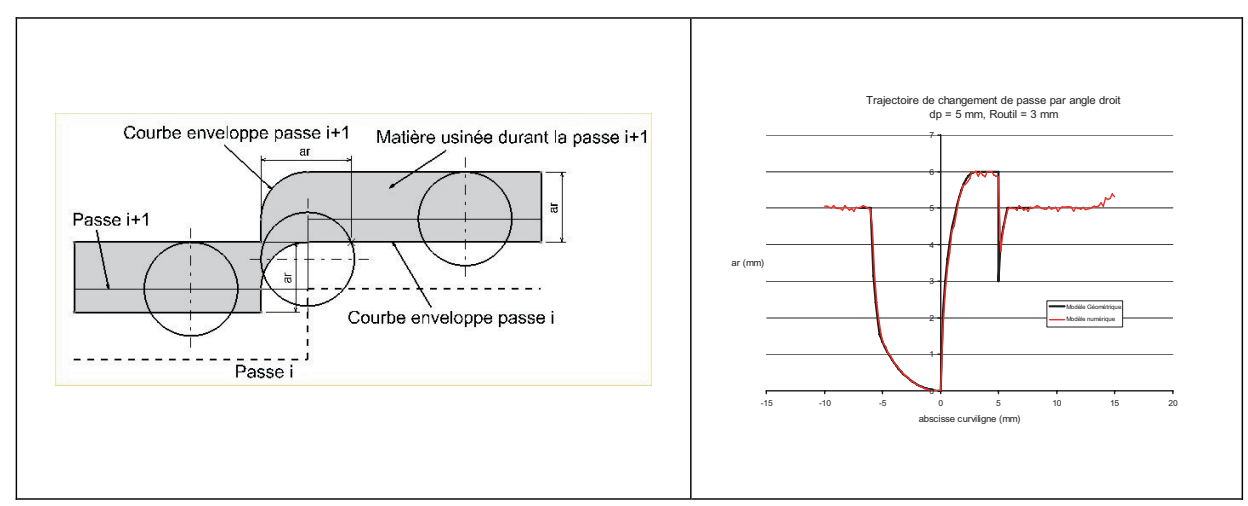

Fig. 2. Évolution de l'engagement radial lors d'un changement de passe.

passes [3,4]. À chaque changement de passe, des discontinuités de trajet en tangence ou en courbure apparaissent (voir Fig. 2). Le tracé de l'engagement radial montre alors de grandes variations lors de leur franchissement $[5,6]$.

Lorsque la zone à usiner présente un profil particulièrement complexe ou comporte des îlots, on considère qu'elle présente plusieurs foyers. Dans ce cas, il n'existe pas de trajet d'usinage permettant d'usiner toute la zone avec une seule plongée dans la matière ou permettant de ne pas repasser avec l'outil sur des portions déjà usinées. Pour rejoindre une zone dépendante d'un foyer non usiné, l'outil doit remonter à une altitude de référence et plonger à nouveau dans la matière; il peut également traverser une partie de la zone en usinant en pleine matière ou il peut rester sur le fond de la pièce sans usiner. Nous verrons par la suite, que ce choix influe à la fois sur le comportement de l'outil et le temps d'usinage.

Afin de faire évoluer les trajets d'usinage, les éditeurs de FAO ont proposé deux nouvelles fonctionnalités.

La première concerne l'adaptation des passes à l'UGV. La trajectoire est cornerisée par l'ajout de rayons de raccordement à chaque discontinuité en tangence du trajet. Cela permet alors de lisser le trajet et de le rendre plus fluide. Mais cette option a aussi une influence sur la variation de l'engagement radial de l'outil et sur la quantité de matière résiduelle. Un trop grand rayon risque d'engendrer des zones non usinées qui peuvent nécessiter une opération de reprise impliquant des passes d'usinage supplémentaires.

La seconde concerne la prise en compte de l'usinage pleine matière grâce à l'adoption d'une stratégie basée sur un motif trochoïdal. Cette nouvelle stratégie doit améliorer le comportement des outils lors de l'usinage de matériaux très durs. Ce type d'outil ne tolère pas un usinage en pleine matière. Cette stratégie privilégie un très faible engagement radial et un fort engagement axial. Par contre, les trajets sont nettement plus longs, ce qui diminue sensiblement la productivité par rapport à un trajet « classique ». Il convient donc de restreindre son emploi aux zones et aux couples outil/matière le nécessitant, c'est-à-dire lors de l'ouverture de poches, le contournement d'îlot en pleine matière ou lors de trajets de liaison entre deux foyers d'une même zone.
Ainsi, le programmeur se trouve face à un nombre important d'options dont il ne connait pas forcément l'usage et les limites. De plus, il ne peut pas estimer l'influence de chaque option sur le comportement de l'outil et de la machine pendant l'usinage. Il convient donc de réaliser une étude du comportement cinématique des machines outils UGV afin de quantifier l'influence de la géométrie du trajet sur la vitesse d'avance réelle de l'outil.

\section{2 Étude du comportement cinématique de la machine outil pendant l'usinage}

En UGV, l'augmentation de la vitesse de coupe engendre naturellement une augmentation de la vitesse d'avance. Les consignes de vitesse sont donc plus importantes. À la suite des publications de Serge Bloch, Vincent Pateloup montre dans sa thèse que la consigne de vitesse d'avance ne peut pas être continuellement atteinte tout au long du parcours d'usinage [7]. Il est donc illusoire d'optimiser la valeur de l'avance à la dent, puisque celle-ci varie fortement, à cause des ralentissements apparaissant durant le suivi du parcours programmé. L'auteur identifie trois phénomènes principaux engendrant des ralentissements :

- la présence de discontinuité en tangence ou en courbure sur le trajet. Théoriquement le passage d'une discontinuité ne peut être fait qu'avec une vitesse nulle. Si la vitesse est plus grande, cela engendre à la fois une plus grande erreur au contour et des vibrations $[8,9]$;

- la diminution du rayon de courbure du trajet. La puissance des axes de déplacement de la machine étant limitée, il n'est pas possible de suivre un arc de cercle de faible rayon à grande vitesse ;

- la valeur du jerk des axes. Plus que l'accélération maximale atteignable, il apparaît que le premier facteur limitant une machine est le jerk. Plus le jerk est faible, plus la machine est souple et lente. À l'opposé, un jerk important permet un franchissement plus rapide des discontinuités car la machine est plus dynamique. Aucune valeur de jerk ne permet de passer une discontinuité sans ralentissement. Un jerk important permet seulement d'atteindre à nouveau la vitesse de consigne rapidement. 


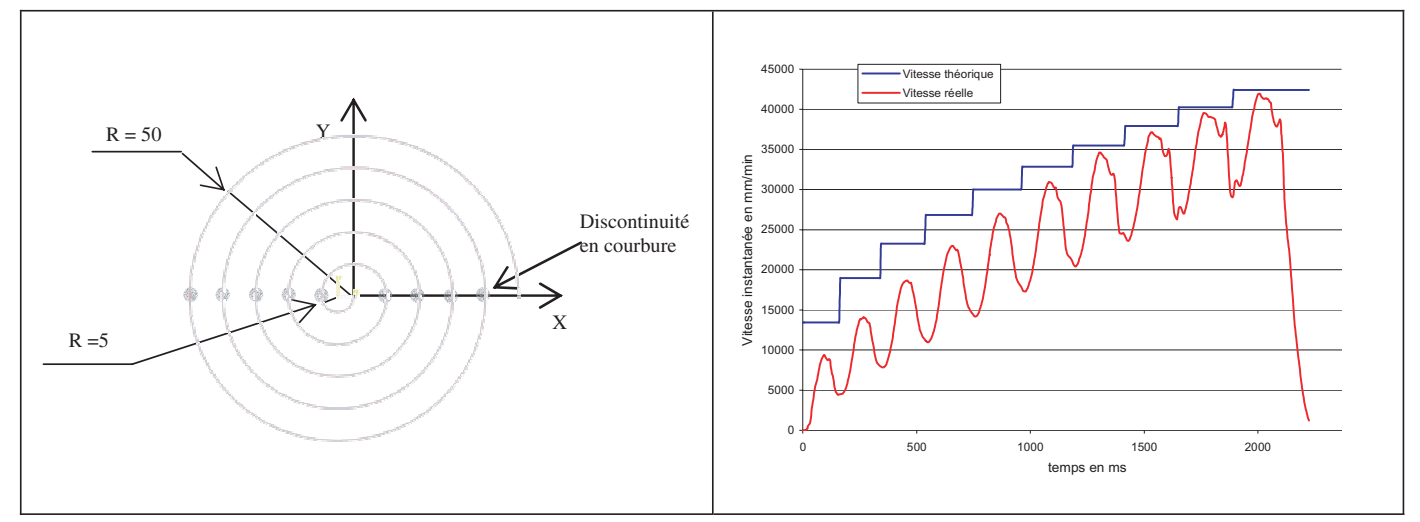

Fig. 3. Évolution de la vitesse réelle en fonction du rayon du trajet.

Tableau 1. Comparaison de machines outils.

\begin{tabular}{|c|c|c|c|}
\hline Comparaison des temps de parcours & Machine outil & Temps & Vitesse moyenne \\
\hline \multirow{9}{*}{ 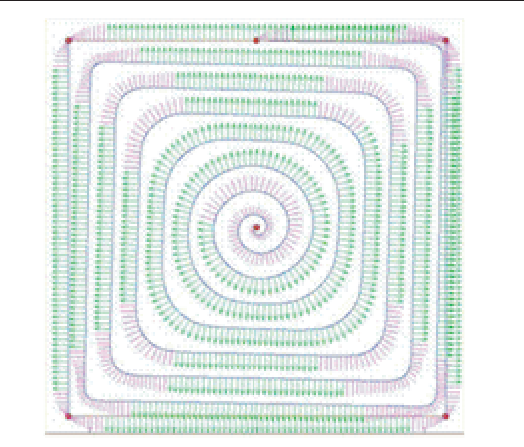 } & Machine A (jerk $10 \mathrm{~m} . \mathrm{s}^{-3}$ ) & $11,8 \mathrm{~s}$ & $7,5 \mathrm{~m} \cdot \mathrm{min}^{-1}$ \\
\hline & Machine A (jerk $20 \mathrm{~m} \cdot \mathrm{s}^{-3}$ ) & $11,5 \mathrm{~s}$ & $7,65 \mathrm{~m} \cdot \mathrm{min}^{-1}$ \\
\hline & Machine A (jerk $30 \mathrm{~m} \cdot \mathrm{s}^{-3}$ ) & $11,3 \mathrm{~s}$ & $7,8 \mathrm{~m} \cdot \mathrm{min}^{-1}$ \\
\hline & Machine B parallèle (jerk 500 m.s ${ }^{-3}$ ) & $9,9 \mathrm{~s}$ & $8,9 \mathrm{~m} \cdot \mathrm{min}^{-1}$ \\
\hline & Machine B parallèle (jerk 1000 m.s ${ }^{-3}$ ) & $9,8 \mathrm{~s}$ & $9 \mathrm{~m} \cdot \mathrm{min}^{-1}$ \\
\hline & Machine B parallèle (jerk 2000 m.s ${ }^{-3}$ ) & $9,76 \mathrm{~s}$ & $9,05 \mathrm{~m} \cdot \mathrm{min}^{-1}$ \\
\hline & Machine B parallèle $\left(V f 20000 \mathrm{~mm} \cdot \mathrm{min}^{-1}\right)$ & $5 \mathrm{~s}$ & $17,6 \mathrm{~m} \cdot \mathrm{min}^{-1}$ \\
\hline & Machine C parallèle & $10,5 \mathrm{~s}$ & $8,4 \mathrm{~m} \cdot \mathrm{min}^{-1}$ \\
\hline & Machine D & $11,5 \mathrm{~s}$ & $7,7 \mathrm{~m} \cdot \mathrm{min}^{-1}$ \\
\hline$V f=10000 \mathrm{~mm} \cdot \mathrm{min}^{-1}$ & Machine E & $8,9 \mathrm{~s}$ & $9,9 \mathrm{~m} \cdot \mathrm{min}^{-1}$ \\
\hline Longueur du trajet $=1470 \mathrm{~mm}$ & Machine $\mathrm{E}\left(V f 20000 \mathrm{~mm} \cdot \mathrm{min}^{-1}\right)$ & $6,15 \mathrm{~s}$ & $14,3 \mathrm{~m} \cdot \mathrm{min}^{-1}$ \\
\hline
\end{tabular}

Pour illustrer ces phénomènes, la trajectoire suivante a été programmée sur plusieurs machines outils. Cette trajectoire est formée de différents arcs de cercle concentriques dont le rayon varie de 5 à $50 \mathrm{~mm}$. La figure 3 montre le relevé de vitesse. Il met en évidence les ralentissements au passage des discontinuités et l'augmentation graduelle de la vitesse pour chaque rayon.

Une autre illustration est présentée dans le tableau 1. Celui-ci compare différentes machines en terme de temps d'usinage. Sur le graphique, les portions de trajet où la vitesse programmée est atteinte sont représentées par des flèches vertes. Cet essai montre que l'augmentation du jerk est le facteur principal de réduction du temps d'usinage. Mais le gain est réduit, lorsque le trajet est continu, car il y a moins de ralentissements.

Ainsi, pour garantir une vitesse d'avance constante, il est nécessaire d'adapter la forme du trajet pour augmenter les rayons de courbure et éliminer les discontinuités en tangence et en courbure. Cette contrainte est d'autant plus importante que la vitesse d'avance programmée est importante et la valeur du jerk faible. Les systèmes de FAO ont tenté de le faire en proposant des fonctions «HSM », qui ajoutent des congés de raccordement sur la trajectoire. Mais il faut remarquer que l'ajout de rayons de raccordement n'est pas toujours performant, car la valeur du rayon doit être suffisamment grande pour que le franchissement de deux discontinuités en courbure et le passage du rayon soient plus rapides que le franchissement d'une discontinuité en tangence. Sur une machine UGV usuelle le rayon minimal doit être supérieur à environ $10 \mathrm{~mm}$, pour que le temps d'usinage soit réduit. Cela peut alors imposer de réduire la distance entre passes pour ne pas laisser de zones non usinées.

Plus généralement, le calcul de trajets lissés est réalisé pour augmenter le rayon de courbure en chaque point du trajet et ainsi la valeur de la vitesse d'avance. Par contre, il faut remarquer que le lissage a aussi tendance à augmenter la longueur du trajet, ce qui réduit le gain final.

En UGV, les constructeurs cherchent à concevoir des machines rigides, pour augmenter le jerk et résoudre le problème du franchissement des discontinuités du trajet. Cette réponse nous paraît insuffisante et l'obtention de trajets continus et lisses semble être une nécessité, car des trajets lisses pourront toujours être plus facilement suivis, quelle que soit la machine.

Il convient maintenant d'étudier le comportement de l'outil en usinage. 
Débit copeau (ccm/min)

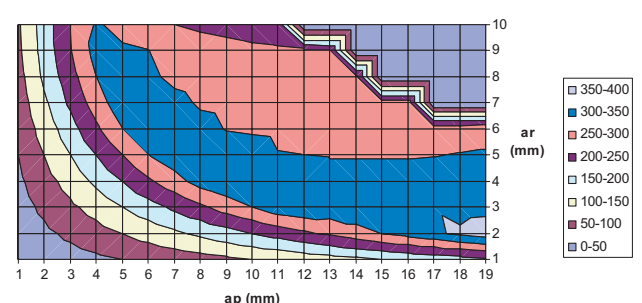

ap (mm)

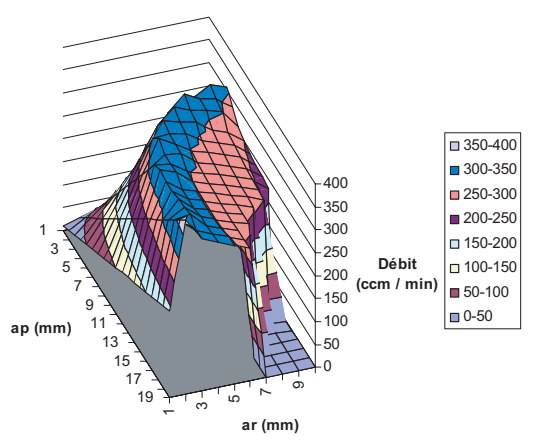

Fig. 4. Évolution du débit copeau en fonction des profondeurs de passe.

\section{3 Étude du comportement de l'outil en usinage}

Nous nous intéressons ici uniquement aux paramètres et aux phénomènes influençant ou étant influencés par le trajet d'usinage. De ce point de vue, les paramètres suivants permettent de caractériser un usinage :

- le mode de coupe : usinage en avalant, en opposition ou mixte, pleine matière autorisée ou non;

- les conditions de coupe : la vitesse de coupe, l'avance à la dent, la profondeur de passe axiale et la profondeur de passe radiale.

Le respect ou non du mode de coupe est une contrainte fondamentale dans le processus, car il impose de modifier les trajectoires. Aussi, est-il nécessaire d'évaluer rapidement si le respect de cette contrainte est fondamental pour l'outil. En effet, un outil supportant un usinage mixte offre plus de libertés dans la construction du trajet et ainsi dans la recherche d'une plus grande productivité.

Lors de la programmation d'une opération d'usinage, il faut spécifier le jeu de conditions de coupe optimal. En ébauche, celui-ci doit produire le débit copeau maximal dans le domaine d'emploi de l'outil. Comme nous l'avons déjà montré, la consigne d'avance à la dent et la consigne d'engagement radial ne sont pas garanties pendant l'usinage. Par contre, la profondeur de passe axiale et la vitesse de coupe sont vérifiées pendant l'usinage.

En fraisage, le choix du jeu de conditions de coupe le plus productif n'est pas aisé, car il y a une interaction entre les quatre paramètres. Le logiciel Coromant Plura de Sandvik permet de l'illustrer sur un cas simple, en calculant le débit copeau pour un couple outil/matière donné. Une fois l'outil sélectionné, le logiciel propose une avance à la dent et une vitesse de coupe pour un jeu de profondeurs de passe axiale et radiale données. La figure 4 représente l'évolution du débit copeau en fonction des profondeurs de passes axiale et radiale, dans le cas de l'usinage d'un aluminium standard avec une fraise de diamètre $10 \mathrm{~mm}$.

Ce graphique montre que le rapport entre la profondeur de passe axiale et la profondeur de passe radiale influence fortement le débit copeau. En particulier, l'usinage en pleine matière n'est pas forcément le plus productif. Il est en effet très sollicitant pour l'outil, dont les dents sont longtemps en contact avec la matière. La vitesse de coupe et l'avance à la dent sont alors réduites pour éviter une usure prématurée et un bris de la dent. De plus, une variation de la profondeur de passe radiale liée à la forme du trajet doit introduire immédiatement une modification de la vitesse d'avance sous peine de rupture de l'outil. Le contrôle de l'engagement radial de l'outil est fondamental dans la recherche d'une grande productivité. Ainsi, il vaut mieux privilégier la profondeur de passe axiale à la profondeur de passe radiale en terme de débit copeau instantané.

En considérant le trajet calculé dans le cas de l'ébauche des moules, il apparaît que ces contraintes ne peuvent être totalement vérifiées. Le contrôle de l'engagement radial est donc primordial. De plus, le système de FAO doit permettre d'adapter les conditions de coupe lors de l'entrée et de la sortie de la matière, ainsi que dans les portions de trajet en pleine matière. Peu de travaux de recherche ont étudié l'influence de ces portions de trajet sur l'usure des outils et leur rupture en fatigue.

En conclusion, la recherche de productivité impose de choisir un jeu de conditions de coupe produisant le plus important débit de copeaux. Mais les conditions de coupe choisies doivent être cohérentes avec deux contraintes importantes : le respect du mode de coupe et la possibilité d'usiner en pleine matière. En effet, ces contraintes sont fondamentales, car elles influencent directement la forme du trajet d'usinage. Un outil autorisant à la fois l'usinage en pleine matière et un mode de coupe mixte offre plus de choix de trajets d'usinage, qu'un outil autorisant uniquement l'usinage en avalant et un engagement radial inférieur à $80 \%$ de son diamètre.

\section{Choix et adaptation des stratégies d'usinage}

\subsection{Expression des contraintes d'usinage}

Lorsque l'on envisage de programmer une opération d'ébauche, il devient nécessaire de définir la classe de problème, pour identifier rapidement la meilleure solution d'usinage. La classe de problème est la conjugaison de l'ensemble des contraintes précédemment énoncées, avec trois niveaux d'exigence : peu contraignante, très contraignante, variation autorisée sur $x \%$. Nous reportons dans le tableau 2 l'ensemble des contraintes étudiées dans cette communication.

La prise en compte du comportement de la machine peut être caractérisée par le tableau 3. Il met en relation le besoin en vitesse et les possibilités de la machine. Plus 
Tableau 2. Contraintes imposées par le couple outil/matière.

\begin{tabular}{|l|l|l|l|}
\hline Conditions d'usinage & \multicolumn{3}{|c|}{ Contrainte } \\
\hline & Oui & Non & $\begin{array}{c}\text { Variation } \\
\%\end{array}$ \\
\hline $\begin{array}{l}\text { Respect du mode de coupe } \\
\text { (avalant/opposition) }\end{array}$ & & & \\
\hline Usinage pleine fraise & & & \\
\hline Contrôle de l'engagement radial & & & \\
\hline Mouvement autorisé sur le fond & & & \\
\hline $\begin{array}{l}\text { Modes d'entrée et de retrait } \\
\text { de la matière }\end{array}$ & & & \\
\hline
\end{tabular}

Tableau 3. Expression du comportement de la machine.

\begin{tabular}{|l|l|l|}
\hline Machine & \multicolumn{2}{|c|}{ Caractéristique } \\
\hline & faible & forte \\
\hline Dynamique des axes & & \\
\hline Vitesse programmée & & \\
\hline
\end{tabular}

la vitesse programmée est importante, plus la dynamique des axes est faible et plus il est nécessaire d'augmenter le rayon de courbure tout au long du trajet et d'éliminer les discontinuités.

La satisfaction simultanée de certaines contraintes est parfois incompatible. En effet, on ne peut pas interdire l'usinage pleine matière ou le mouvement sur le fond si les retraits matière sont interdits, dans le cas d'usinage d'une poche à plusieurs foyers.

De même, la satisfaction d'une contrainte rend caduque ou entraîne naturellement la satisfaction d'autres contraintes. Par exemple, l'autorisation de l'usinage pleine matière permet une grande variation de l'engagement radial.

Il n'est pas simple de procéder au calcul d'un trajet respectant l'ensemble des contraintes. En particulier, il n'existe pas un seul type de trajet capable de respecter toutes les contraintes dans tous les cas de figure. Il convient alors d'identifier le plus productif, c'est-à-dire le plus rapide, tout en ne dépassant pas le taux de charge à l'outil engendrant sa rupture ou son usure prématurée.

L'expérience montre qu'il y a peu de libertés d'optimisation, lorsque les contraintes imposées par l'outil sont très exigeantes. En particulier, l'usinage systématique en avalant, l'interdiction d'usinage pleine matière et la contrainte de respecter la valeur de la profondeur de passe radiale ne sont garantis que pour un seul type de trajectoire : l'usinage concentrique suivant une spirale. Il est alors nécessaire de libérer certaines contraintes en autorisant des variations autour de la consigne pour gagner en productivité.

\subsection{Choix et paramétrage de la stratégie d'usinage}

Nous nous intéressons par la suite au choix d'une stratégie respectant le mode de coupe.
Le facteur primordial du choix de la stratégie d'usinage concerne la variation d'engagement radial. Si la variation autorisée est faible, alors seules des stratégies de type « concentrique» (voir Fig. 5) peuvent le garantir. Par contre, dès que cette contrainte est levée, il est possible d'utiliser une stratégie plus classique comme l'usinage de type « hélicoïdal ». On peut alors profiter de la liberté de variation pour réduire le temps d'usinage (voir Fig. 5).

L'usinage en pleine fraise constitue la seconde contrainte importante. Interdire cet usinage peut obliger à ouvrir les poches en plusieurs endroits, engendrant des mouvements de changement de zone et d'entrée dans la matière. Par contre, si la contrainte n'est pas posée, il est possible de passer d'une zone à l'autre par un mouvement direct plus court.

La programmation actuelle des modes d'usinage de type « hélicoïdal » ne permet pas de gérer la valeur de l'engagement radial maximal. Par contre, on peut lisser ou réduire la variation d'engagement radial en utilisant les fonctions « HSM »d'ajout de rayon de congés.

En ce qui concerne la vitesse réelle d'usinage, il faut bien définir si l'usinage est considéré à grande vitesse du point de vue de la machine. L'augmentation de la valeur des rayons de courbure et l'élimination des discontinuités sont à appliquer essentiellement lorsque la vitesse d'avance programmée est très importante. Lorsque la machine n'est pas très dynamique, il est inutile d'ajouter des rayons de raccordement, sauf si leur valeur est suffisamment grande pour ne pas produire de ralentissements. Cela impose alors des contraintes sur la distance entre passes, selon la valeur du diamètre de l'outil, pour garantir que toute la zone est usinée.

\section{4 Étude d'un cas d'application}

Considérons le cas d'usinage d'une poche comportant un îlot (voir Fig. 5). Ce type d'usinage pose le problème du contournement de l'îlot. En effet, la fraise doit nécessairement usiner en pleine matière. On peut alors se poser le problème de trouver le trajet qui sollicite le moins possible la fraise sans pour autant induire un temps d'usinage trop grand. Plusieurs stratégies d'usinage sont envisageables, nous présentons deux très différentes. Pour chaque trajet, un code de couleur est utilisé pour représenter les variations de l'engagement radial, calculées en chaque point du trajet.

Les stratégies proposées sont fondamentalement différentes et présentent des résultats aussi très différents au niveau du comportement de l'outil et de la machine pendant l'usinage. La première solution est un usinage concentrique qui permet de contrôler très précisément l'engagement radial. Sur la figure précédente, on constate que l'outil usine très peu en pleine matière. Le mode de coupe est toujours respecté et les conditions de coupe de l'outil peuvent être garanties tout le long du trajet. Une première entrée dans la matière est réalisée dans la zone la plus grande. Puis, le logiciel génère des passes concentriques pour ouvrir la poche. Lorsqu'une zone est terminée, l'outil est positionné au milieu de la zone suivante 


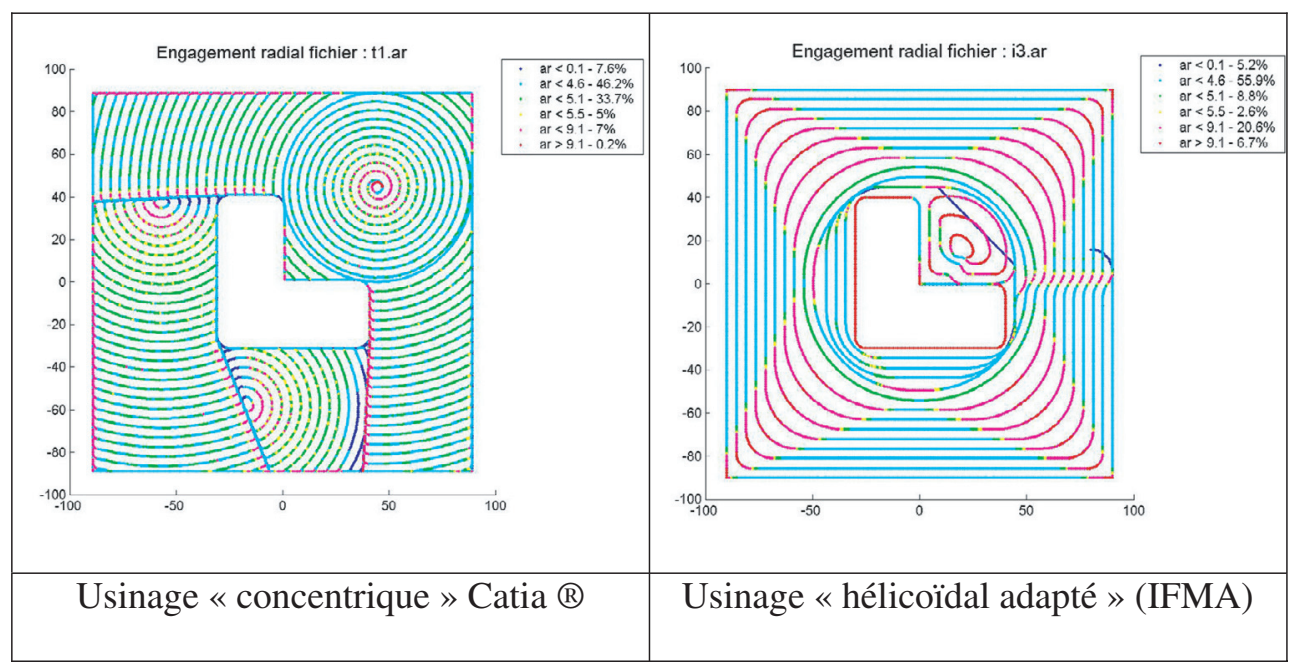

Fig. 5. Comparaison de parcours d'usinage pour l'évidement d'une poche avec îlots.

Tableau 4. Comparaison des performances.

\begin{tabular}{|l|c|l|c|}
\hline Distance entre passes & $4,5 \mathrm{~mm}$ & Distance entre passes & $4,5 \mathrm{~mm}$ \\
\hline Distance entre passes maximale & néant & Distance entre passes maximale & $9,0 \mathrm{~mm}$ \\
\hline Nombre de foyers & 1 & Nombre de foyers & 1 \\
\hline Usinage pleine matière & non & Usinage pleine matière & Oui \\
\hline Longueur de trajectoires & $13708 \mathrm{~mm}$ & Longueur de trajectoires & $7745 \mathrm{~mm}$ \\
\hline Temps d'usinage Catia & $12 \mathrm{~min} 55 \mathrm{~s}$ & Temps d'usinage Catia & $6 \mathrm{~min} 42 \mathrm{~s}$ \\
\hline Temps d'usinage réel & $13 \mathrm{~min} 57 \mathrm{~s}$ & Temps d'usinage réel & $7 \mathrm{~min} 08 \mathrm{~s}$ \\
\hline Vitesse moyenne & $1 \mathrm{~m} \cdot \mathrm{min}^{-1}$ & Vitesse moyenne & $1 \mathrm{~m}^{-m_{i n}}{ }^{-1}$ \\
\hline Temps d'usinage réel UGV & $4 \mathrm{~min} 15 \mathrm{~s}$ & Temps d'usinage réel UGV & $1 \mathrm{~min} 01 \mathrm{~s}$ \\
\hline Vitesse moyenne & $3,2 \mathrm{~m} \cdot \mathrm{min}^{-1}$ & Vitesse moyenne & $7,6 \mathrm{~m} . \mathrm{min}^{-1}$ \\
\hline \multicolumn{2}{|c|}{ Usinage « concentrique $»$} & Usinage « hélicoïdal adapté » \\
\hline
\end{tabular}

pour procéder à l'ouverture. Le code de couleur montre que l'engagement radial maximal est obtenu à l'ouverture des zones et à l'entrée de l'outil dans la matière à chaque passe.

À l'opposé la seconde solution est directement issue d'un usinage de type hélicoïdal, adaptée pour tenir compte de l'engagement radial et du comportement cinématique de la machine. Un premier usinage est réalisé pour ouvrir la poche jusqu'à obtenir un profil proche du profil extérieur décalé. Pour cela, l'îlot est contourné par un usinage en pleine matière. Puis le trajet s'écarte pour approcher le profil extérieur de la poche. Cette solution engendre une variation importante de l'engagement radial de l'outil dans les coins et dans les changements de passe (voir code de couleur). Mais cette variation est contrôlée par un paramètre d'engagement maximal choisi égal à $9 \mathrm{~mm}$ pour un outil de $10 \mathrm{~mm}$ de diamètre.

On constate alors que les deux stratégies proposées produisent des solutions très différentes. La différence principale se situe au niveau du contrôle de l'engagement radial plus ou moins restrictif. Cette latitude permet alors de calculer des trajets plus rapides qui optimisent cinématiquement le comportement de la machine outil. Le tableau 4 montre le gain apporté sur le temps d'usinage. Dans un premier temps, la longueur du trajet est nettement plus grande dans le premier cas. De plus, l'usinage sur un centre d'usinage Huron Kx15 avec une vitesse d'avance de $1000 \mathrm{~mm} \cdot \mathrm{min}^{-1}$ montre que les deux trajets produisent une vitesse moyenne réelle similaire. Par contre, si on multiplie par 10 la valeur de la vitesse d'avance programmée, alors les deux vitesses moyennes réelles ne sont plus similaires. Ainsi, la forme du trajet est un facteur de ralentissement important.

\section{Conclusion}

Cette communication présente une réflexion sur l'influence de la forme du trajet d'usinage sur le comportement de la cellule d'usinage, et ainsi sur la nécessaire cohérence entre les différents systèmes de la chaîne de fabrication : CAO, FAO, CN, machine outil et outil. Elle aborde le cas d'application de l'ébauche UGV des pièces de forme complexe.

L'augmentation des exigences de performance a entraîné le développement de nombreux algorithmes de calcul de trajet différents et difficiles à paramétrer. Il convient alors de proposer en accompagnement du 
programmeur des outils de diagnostic qui lui permettent d'identifier rapidement la meilleure solution de programmation et de vérifier la satisfaction des contraintes par le trajet.

Après analyse des comportements de l'outil et de la machine en usinage, nous montrons que les deux contraintes principales se situent au niveau de l'engagement de la fraise et du suivi de trajectoires.

Pour procéder à une programmation efficace, l'utilisateur doit définir précisément sa classe de problème en évaluant les contraintes imposées par l'outil et sur la machine. Nous montrons que la libération de certaines contraintes est facteur important de gain en productivité.

De même utiliser des options «UGV », lorsque cela n'est pas utile, peut engendrer un effet néfaste : ralentissement et apparition de zones non usinées.

À la suite de ces travaux, nous pouvons envisager de proposer une nouvelle approche pour le calcul de trajet d'usinage basée sur la spécification du besoin et la certification du trajet.

\section{Références}

[1] A. Hatna, R.J Grieve, P. Brommhead, Automatic CNC milling of pockets: geometric and technological issues, Computer Integrated Manufacturing Systems 11 (1998) 309-330
[2] C.A.H. Lambregts, F.L.M. Delbressine, W.A.H. De Vries, A.C.H. van der Wolf, An efficient automatic tool path generator for $21 / 2 \mathrm{D}$ free-form pockets, Computer in Industry 29 (1996) 151-157

[3] S.C. Park, B.K. Choi, Uncut free pocketing tool path generation using pair-wise offset algorithm, Computer Aided Design 33 (2001) 739-746

[4] V. Pateloup, E. Duc, P. Ray, Pocketing optimization for HSM : Geometry tool path and interpolation mode influence on dynamic machine tool behaviour, Machine Engineering 3 (2003) 127-138

[5] J. Klopayan, Y.S. Lee, Material engagement analysis of different end mills for adaptive feedrate control in milling Processes, Computer in Industry 47 (2002) 55-79

[6] H. Chanal, E. Duc, P. Ray, Material removal analysis for pocket machining, 5th International Conference on Integrated Design and Manufacturing in Mechanical Engineering, 2004, Actes sur CRDROM, Bath (UK)

[7] V. Pateloup, Amélioration du comportement cinématique des machines outils UGV, Application au calcul de trajets d'évidement de poches, Thèse de l'Université Blaise Pascal, juillet 2005

[8] S. Bloch, E. Deneuville, L. Tan, Inovate feedrate optimisation technique, $3^{\mathrm{e}}$ congrès international sur la coupe des métaux et l'usinage grande vitesse, Metz, juin 2001

[9] K. Erkorkmaz, Y. Atlintas, High speed CNC system design, Part 1: jerk limited trajectory generation and quintic spline interpolation, Int. J. Machine Tool Manufacture 41 (2001) 1323-1345 